\title{
Segurança alimentar em famílias com crianças matriculadas em creches públicas do Estado da Paraíba, Brasil
}

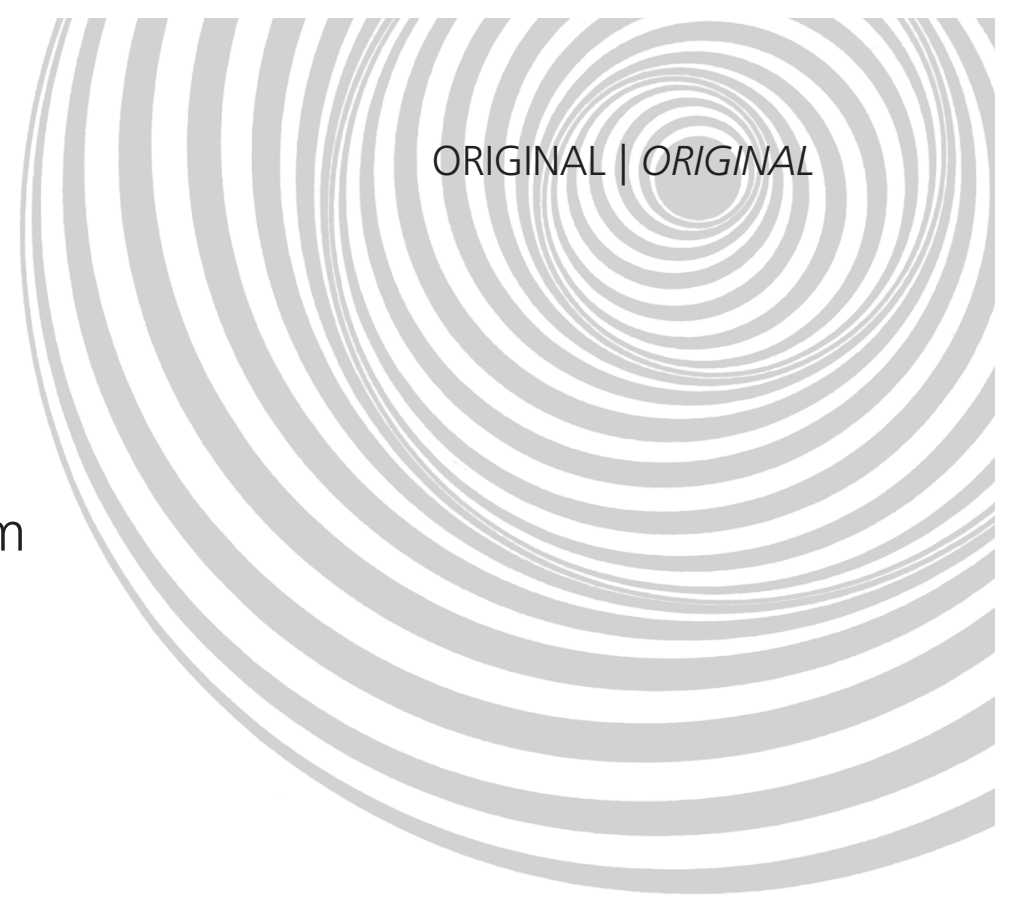

\author{
Food security in families with children \\ attending public daycare centers in \\ the State of Paraíba, Brazil
}

Dixis FIGUEROA PEDRAZA

Daiane de QUEIROZ2

Tarciana Nobre de MENEZES 3

RE S U M O

\section{Objetivo}

Estimar a prevalência de insegurança alimentar e examinar fatores associados em famílias com crianças matriculadas em creches públicas do Estado da Paraíba.

\section{Métodos}

Estudo transversal com informações familiares sobre segurança alimentar, estado nutricional de crianças, características materno-infantis e condições socioeconômicas. Calculou-se tamanho de amostra de 365 indivíduos pelo procedimento para descrição da proporção. O estado nutricional das crianças foi analisado considerando os índices estatura/idade, peso/estatura e peso/idade. A (in)segurança alimentar foi avaliada por meio da Escala Brasileira de Insegurança Alimentar. A associação entre as variáveis independente e dependente foi estimada aplicando-se o teste Qui-quadrado e modelo multivariado.

\section{Resultados}

A insegurança alimentar foi caracterizada em 62,0\% das famílias, predominando as condições de insegurança leve (33,4\%). As variáveis renda familiar per capita, benefício do Programa Bolsa Família, tempo de aleitamento materno total e tempo de aleitamento materno exclusivo apresentaram associação inversa significante com a insegurança alimentar.

1 Universidade Estadual da Paraíba, Departamento de Enfermagem, Programa de Pós-Graduação em Saúde Pública. Av. das Baraúnas, 351, Bodocongó, 58429-500, Campina Grande, PB, Brasil. Correspondência para/Correspondence to: D FIGUEROA PEDRAZA. E-mail: <dixisfigueroa@gmail.com>.

2 Centro de Ensino Superior e Desenvolvimento, Faculdade de Ciências Médicas de Campina Grande. Campina Grande, PB, Brasil.

3 Universidade Estadual da Paraíba, Departamento de Fisioterapia, Programa de Pós-Graduação em Saúde Pública. Campina Grande, PB, Brasil. 
518 | D FIGUEROA PEDRAZA et al.

\section{Conclusão}

A renda familiar per capita foi o indicador que melhor discriminou a insegurança alimentar, o que sugere o comprometimento do acesso à alimentação e a importância da transferência de renda focalizada.

Termos de indexação: Creches. Criança. Estado nutricional. Fatores socioeconômicos. Insegurança alimentar. Prevalência.

\section{A B S T R A C T}

\section{Objective}

This work aims to evaluate the prevalence of food insecurity and associated factors in families with children enrolled in daycare centers.

\section{Methods}

This cross-sectional study collected data on the family's food security and socioeconomic statuses, nutritional status of the children, and mother and child characteristics. A sample size of 365 individuals was calculated by procedure for describing the proportion. The children's nutritional status was determined by analyzing their height-for-age, weight-for-height, and weight-for-age. Food (in)security was determined by the Brazilian Food Insecurity Scale. The association between the independent and dependent variables was estimated by the Chi-square test and multivariate model.

\section{Results}

Most families (62.0\%) experienced food insecurity, especially mild food insecurity (33.4\%). The variables 'family per capita income', welfare (Bolsa Família Program) status, and total exclusive and nonexclusive breastfeeding time were inversely associated with food insecurity.

\section{Conclusion}

The indicator that best distinguished food insecurity was family per capita income, suggesting poor access to food and the importance of targeted welfare.

Indexing terms: Child day care centers. Child. Nutritional status. Socioeconomic factors. Food insecurity. Prevalence.

\section{N T R O D U Ç Ã O}

A inclusão da alimentação entre os direitos constitucionais consolidou a segurança alimentar e nutricional como uma questão de cidadania. Assim, a segurança alimentar e nutricional deve ser visualizada como questão coletiva e de um conjunto de necessidades, com base em práticas alimentares promotoras de saúde que respeitem a diversidade cultural e que sejam social, econômica e ambientalmente sustentáveis ${ }^{1}$. A insegurança alimentar, determinada, principalmente, pela pobreza e pelas desigualdades sociais, tem sido tema relevante na literatura nacional e internacional nas áreas de Saúde, Nutrição e das Ciências Sociais, em geral, enfocando a sua ocorrência nos diferentes grupos da população, suas repercussões e seus determinantes ${ }^{2}$.
Conhecer a prevalência de insegurança alimentar e fatores associados é de grande importância para a avaliação das condições de vida e, consequentemente, para o planejamento de programas e políticas públicas de caráter preventivo, promoção da saúde e combate à fome ${ }^{3,4}$. Com isso, tornou-se imperiosa a busca de indicadores da situação de segurança/insegurança alimentar que possibilitem o acompanhamento da sua evolução, a avaliação de propostas de intervenção e que permitam comparabilidade ao longo do tempo e segundo as diversas regiões².

Existem cinco métodos comumente utilizados para mensurar a segurança alimentar ${ }^{5}$. Quatro são métodos indiretos baseados nas repercussões de estados de insegurança alimentar no consumo de alimentos ou no estado nutricional: 
i) método da Organização das Nações Unidas para Agricultura e Alimentação; ii) pesquisas de despesas familiares, iii) avaliação de consumo de alimentos; e iv) avaliação antropométrica. O único método para a medida direta de segurança alimentar é representado por uma escala fundamentada na experiência/percepção de insegurança alimentar ${ }^{2,5}$.

A escala proposta por Radim et al. ${ }^{6}$ vem sendo aplicada em diversos países para dimensionar a magnitude da insegurança alimentar ${ }^{3}$. Embasados nesse instrumento, pesquisadores de várias instituições brasileiras validaram um método para a avaliação da segurança alimentar em população brasileira, denominada Escala Brasileira de Insegurança Alimentar (EBIA $)^{7}$. Essa escala tem sido reconhecida como indicador sensível para detectar famílias em risco de insegurança alimentar ${ }^{8}$.

Os primeiros resultados de base populacional e representatividade nacional com a utilização da EBIA foram obtidos na Pesquisa Nacional por Amostra de Domicílios (PNAD), realizada em 2004. Segundo a pesquisa, $34,8 \%$ dos domicílios brasileiros sofrem com algum grau de insegurança alimentar, apresentando variações regionais, com destaque para o Norte e o Nordeste do País, que apresentaram as piores condições ${ }^{8}$.

Os estudos sobre segurança alimentar devem considerar nas suas análises grupos populacionais vulneráveis que, por sua condição biológica, são mais suscetíveis à ingestão deficitária de alimentos. Nesse contexto, destacam-se as gestantes, mães que estejam amamentando, crianças e idosos. As crianças nos primeiros anos de vida são as mais vulneráveis à insegurança alimentar e aos riscos nutricionais 9 .

Nesse sentido, o presente estudo teve por objetivo estimar a prevalência de insegurança alimentar e examinar fatores associados em famílias com crianças matriculadas em creches públicas do Estado da Paraíba.

\section{M É T O D OS}

Trata-se de um estudo epidemiológico de delineamento transversal, que investigou famílias com crianças menores de cinco anos assistidas em creches cadastradas na Secretaria de Estado do Desenvolvimento Humano do Governo da Paraíba. No contexto familiar, a definição do grupo populacional esteve baseada na vulnerabilidade socioeconômica e na presença de crianças menores de cinco anos. Assim, enfatizam-se a conjuntura da desigualdade social na insegurança alimentar assim como a importância de indicadores do estado nutricional de crianças na medição da segurança alimentar.

São 45 creches situadas geralmente em áreas carentes dos 8 municípios paraibanos beneficiados: João Pessoa (30 creches), Campina Grande (9 creches), além das cidades de Areia, Bayeux, Mamanguape, Itaporanga, Soledade e Umbuzeiro (cada uma delas com uma creche). O universo de estudo é de 4 mil crianças beneficiadas, entre 6 e 72 meses de idade: aproximadamente 2800 no município de João Pessoa, 750 no município de Campina Grande e 450 nos outros municípios.

Foi selecionada uma amostra probabilística em duas etapas. Para garantir a representatividade dos municípios, o sistema de referência para a primeira etapa de amostragem foi ordenado segundo estratos (João Pessoa, Campina Grande, outros municípios), o que possibilita a obtenção de um tamanho amostral apropriado para cada estrato. Considerou-se também o porte da creche, isto é, o número de crianças por creche. Na segunda etapa, as crianças a serem avaliadas foram sorteadas das 14 creches selecionadas, de forma aleatória, na primeira etapa. A opção para determinar o tamanho da amostra do estudo foi pelo procedimento para descrição da proporção ${ }^{10}$. Utilizou-se a fórmula $\mathrm{n}=\frac{\mathrm{N} * \mathrm{Z}_{\alpha}^{2} * p^{*} q}{\mathrm{~d}^{2}(\mathrm{~N}-1)+\mathrm{Z}_{\alpha}^{2} p^{*} q} ，$ sendo $\mathrm{N}$ o total da população, $Z \alpha^{2}=1,96^{2}$ (quando o Intervalo de Confiança é de $95 \%$ ), p a proporção esperada, $q=1-p, d$ a precisão arbitrária (erro de 
estimação). Considerou-se $p=10,5 \%$ (crianças com deficit de estatura no Brasil ${ }^{11}$ ) e $d=3 \%$, totalizando 365 crianças que foram selecionadas por sorteio simples no momento do trabalho de campo.

Os procedimentos para a coleta dos dados foram estabelecidos considerando o seguinte modelo de classificação das variáveis independentes do estudo:

- características biológicas das crianças: sexo, idade, peso/idade, estatura/idade, peso/ estatura;

- morbidade e amamentação das crianças: hospitalização nos 12 meses anteriores à entrevista, presença de morbidade nos 15 dias anteriores à entrevista (baseada na referência materna sobre a ocorrência de diarreia, febre, vômitos, tosse e verminose), amamentação (sim ou não), tempo de amamentação total, tempo de amamentação exclusiva;

- antecedentes maternos: idade, estatura/ idade, Índice de Massa Corporal (IMC), cuidados durante a gravidez baseados em condições associadas à gravidez (orientação sobre a amamentação, suplementação com sulfato ferroso, vacina antitetânica, número de consultas de pré-natal, uso de cigarro, uso de álcool);

- condições socioeconômicas: tipo de casa, número de cômodos do domicílio, número de pessoas no domicílio, renda familiar per capita, benefício do Programa Bolsa Família;

Os dados socioeconômicos foram obtidos por meio da ficha da criança, documento obrigatório que informa o perfil das crianças beneficiárias. As informações sobre morbidade e amamentação da criança, cuidados durante a gravidez e benefício do Programa Bolsa Família, foram obtidas por meio do questionário pré-testado aplicado às mães das crianças.

Para a avaliação antropométrica, o comprimento (crianças menores de 24 meses), a estatura (crianças de 25-72 meses), o peso e a estatura da mãe foram obtidos por meio de equipamentos e técnicas padronizadas. Foram seguidos os proce- dimentos recomendados pela World Health Organization $(\mathrm{WHO})^{12}$.

Para a avaliação do estado nutricional das crianças, foram considerados os índices peso/idade, comprimento/idade (estatura/idade) e peso/comprimento (peso/estatura), que foram comparados aos padrões de crescimento infantil da Organização Mundial da Saúde (OMS) ${ }^{13}$, por meio do programa WHO Anthro 2005, versão beta (WHO Anthro, Genebra, Suíça). Foram consideradas com deficit nutricional todas as crianças que apresentaram índices $<-2$ escores-Z do valor mediano da população de referência. Os índices peso/idade e peso/estatura $>+2$ escores- $Z$ do valor mediano da população de referência foram considerados para indicar peso elevado para a idade e sobrepeso/obesidade, respectivamente.

Para a avaliação do estado nutricional das mães, foram considerados os índices estatura/idade e IMC. A baixa estatura materna foi definida pelo ponto de corte $155 \mathrm{~cm}^{14,15}$. Para o IMC, foram utilizados os pontos de corte indicados pela OMS 11

A avaliação da segurança alimentar foi realizada por meio da EBIA, instrumento com 15 perguntas centrais fechadas, com opções de resposta sim ou não, sobre a experiência nos últimos três meses relacionada à alimentação. Inclui desde a preocupação de que a comida possa acabar até a vivência de passar um dia todo sem comer ${ }^{3}$.

As famílias foram classificadas em quatro categorias de segurança alimentar, considerando-se a quantificação do total de respostas afirmativas na escala16,17: (a) Segurança Alimentar para 0 resposta positiva; (b) Insegurança Alimentar Leve para 1-5 respostas positivas; (c) Insegurança Alimentar Moderada para 6-10 respostas positivas; (d) Insegurança Alimentar Grave para 11-15 respostas positivas.

Os dados foram codificados e digitados em planilhas do programa Excel (Microsoft Inc., Estados Unidos), por dois digitadores independentes para verificação e correção de erros de digitação. O aplicativo Validate do programa Epi 
Info v. 6.04b (WHO/CDC, Atlanta, Estados Unidos) foi usado para analisar a consistência dos dados. Para verificar a existência de associação entre a insegurança alimentar e as variáveis independentes do modelo de estudo, foi utilizado o teste Qui-quadrado de Pearson. O nível de significância estatística considerado foi de $5 \%(p<0,05)$. Em seguida, realizou-se análise multivariada em níveis de acordo com as categorias de classificação das variáveis, ajustando os modelos pelas variáveis associadas significativamente ao nível de $5 \%$. As variáveis que permaneceram associadas em cada nível constituíram o modelo hierárquico final. As análises não foram ajustadas por sexo e por idade da criança por não apresentarem diferença significativa no modelo univariado $(p>0,05)$.

A confiabilidade ou consistência interna da EBIA foi analisada por meio do coeficiente $\alpha$-Cronbach, utilizando como valores aceitáveis resultados acima de 0,85. As análises estatísticas foram realizadas por meio do programa Statistical Package for the Social Sciences (SPSS) for Windows versão 17.0 (SPSS Inc., Chicago, Estados Unidos).

Os resultados do presente estudo fazem parte do projeto aprovado pelo Comitê de Ética em Pesquisa da Universidade Estadual da Paraíba, Protocolo n 4233000013307, intitulado "Segurança Alimentar e Nutricional de famílias socioeconomicamente vulneráveis do estado da Paraíba, Brasil: explorando associações entre medidas essenciais de segurança alimentar". No momento da entrevista, foi apresentado o Termo de Consentimento Livre e Esclarecido, no qual o entrevistado assentava a concordância em participar da pesquisa, após esclarecimentos quanto aos procedimentos a serem empregados, a garantia de sigilo das informações prestadas e a possibilidade de declinar a participação.

\section{RES U LTA D O S}

Da amostra de 365 crianças, registrou-se um total de 33 perdas $(9,04 \%)$, ocasionadas pelo não comparecimento das mães ou pessoas res- ponsáveis pelas crianças no momento da coleta de dados ou por questionários de EBIA incompletos. A fim de analisar a possibilidade de viés da amostra, foi comparada a média de idade e a proporção por sexo da amostra final com a amostra das crianças que foram perdidas, não diferindo entre os dois grupos. Isso permite extrapolar os resultados obtidos para o total da população. 0 teste estatístico $\alpha$-Cronbach indicou a consistência e a confiabilidade dos resultados, com validade psicométrica alta $(0,9206)$.

\section{Caracterização geral e condições socioeconômicas das famílias}

A amostra foi constituída por $54,2 \%$ de crianças do sexo masculino e $62,3 \%$ de crianças entre 37 e 60 meses de idade. Os indicadores nutricionais infantis expressaram deficit de estatura e sobrepeso/obesidade de 7,5\% e 7,2\%, respectivamente. Em relação aos dados de morbidade e de amamentação das crianças, $68,1 \%$ delas apresentaram sintomas de infecção, 68,0\% foram hospitalizadas no ano anterior à entrevista, 40,4\% foram amamentadas de forma exclusiva por seis meses ou mais e $27,7 \%$ foram amamentadas por 24 meses ou mais.

Em relação aos dados maternos, 6,9\% das mães tinham idade inferior a 20 anos, sendo alta a prevalência de deficit de estatura $(39,8 \%)$ e de sobrepeso/obesidade (37,9\%). Em relação às condições socioeconômicas, a maior parte das famílias morava em casa própria, de quatro cômodos ou mais, com menos de seis pessoas. A renda familiar per capita teve a maior representação na categoria inferior (<1/2 salário-mínimo): 266 casos do total da amostra. O recebimento do beneficio do Programa Bolsa Família foi observado em $40,1 \%$ das famílias.

Considerando apenas os domicílios com insegurança alimentar, 53,8\% das crianças eram do sexo masculino, $63,8 \%$ tinham entre $37-60$ meses, 7,5\% apresentavam desnutrição crônica e 7,5\% sobrepeso/obesidade. Em relação aos dados maternos, 8,5\% tinham menos de 20 anos, 
522 D FIGUEROA PEDRAZA et al.

Tabela 1. Prevalência de (in)segurança alimentar de famílias com crianças assistidas em creches segundo características biológicas das crianças, morbidade e amamentação das crianças, antecedentes maternos e condições socioeconômicas. Paraíba (PE), 2008.

Continua

\begin{tabular}{|c|c|c|c|c|c|c|}
\hline \multirow{2}{*}{ Variáveis } & \multirow{2}{*}{ Total $(n=332)$} & \multirow{2}{*}{ Segurança alimentar (\%) } & \multicolumn{3}{|c|}{ Insegurança alimentar (\%) } & \multirow{2}{*}{$p$} \\
\hline & & & Leve & Moderada & Grave & \\
\hline \multicolumn{7}{|l|}{ Características biológicas das crianças } \\
\hline Sexo & & & & & & 0,474 \\
\hline Masculino & 180 & 38,9 & 30,0 & 18,9 & 12,2 & \\
\hline Feminino & 152 & 36,8 & 37,5 & 14,5 & 11,2 & \\
\hline Idade & & & & & & 0,567 \\
\hline 6-12 meses & 11 & 18,2 & 45,5 & 18,2 & 18,2 & \\
\hline 13-36 meses & 114 & 42,1 & 35,1 & 14,0 & 8,8 & \\
\hline $37-60$ meses & 207 & 36,7 & 31,9 & 18,4 & 13,0 & \\
\hline Peso/ldade & & & & & & 0,878 \\
\hline Baixo peso para idade & 7 & 28,6 & 42,9 & 14,3 & 14,3 & \\
\hline Peso adequado para idade & 311 & 37,9 & 33,1 & 16,7 & 12,2 & \\
\hline Peso elevado para idade & 14 & 42,9 & 35,7 & 21,4 & 0,0 & \\
\hline Estatua/ldade & & & & & & 0,654 \\
\hline Baixa estatura & 25 & 28,0 & 44,0 & 16,0 & 12,0 & \\
\hline Estatura adequada & 307 & 38,8 & 32,6 & 16,9 & 11,7 & \\
\hline Peso/Estatura & & & & & & 0,235 \\
\hline Baixo peso & 6 & 16,7 & 16,7 & 33,3 & 33,3 & \\
\hline Peso adequado & 302 & 38,1 & 32,8 & 17,2 & 11,9 & \\
\hline Sobrepeso/obesidade & 24 & 41,7 & 45,8 & 8,3 & 4,2 & \\
\hline \multicolumn{7}{|l|}{ Morbidade e amamentação das crianças } \\
\hline Hospitalização nos últimos 12 meses & & & & & & 0,443 \\
\hline Sim & 226 & 35,0 & 35,4 & 17,3 & 12,4 & \\
\hline Não & 106 & 44,3 & 29,2 & 16,0 & 10,4 & \\
\hline Presença de morbidade nos últimos 15 dias & & & & & & 0,565 \\
\hline Sim & 276 & 38,4 & 32,2 & 16,7 & 12,7 & \\
\hline Não & 56 & 35,7 & 39,3 & 17,9 & 7,1 & \\
\hline Amamentação & & & & & & 0,869 \\
\hline $\operatorname{sim}$ & 316 & 38,3 & 33,5 & 16,5 & 11,7 & \\
\hline Não & 14 & 28,6 & 28,6 & 28,6 & 14,3 & \\
\hline Sem informação & 2 & 50,0 & 50,0 & 0,0 & 0,0 & \\
\hline Tempo de amamentação total & & & & & & 0,006 \\
\hline$<24$ meses & 236 & 33,9 & 39,8 & 14,0 & 12,3 & \\
\hline$\geq 24$ meses & 92 & 47,8 & 17,4 & 23,9 & 10,9 & \\
\hline Sem informação & 4 & 50,0 & 25,0 & 25,0 & 0,0 & \\
\hline Tempo de amamentação exclusivo & & & & & & 0,046 \\
\hline$<6$ meses & 192 & 32,3 & 39,1 & 17,2 & 11,5 & \\
\hline$\geq 6$ meses & 134 & 46,3 & 26,1 & 16,4 & 11,2 & \\
\hline Sem informação & 6 & 33,3 & 16,7 & 16,7 & 33,3 & \\
\hline \multicolumn{7}{|l|}{ Antecedentes maternos } \\
\hline Idade (anos) & & & & & & 0,024 \\
\hline$\geq 30$ & 118 & 45,8 & 32,2 & 16,9 & 5,1 & \\
\hline$<30-\geq 25$ & 102 & 38,2 & 30,4 & 18,6 & 12,7 & \\
\hline$>25-\geq 20$ & 83 & 28,9 & 39,8 & 16,9 & 14,5 & \\
\hline$<20$ & 23 & 26,1 & 30,4 & 8,7 & 34,8 & \\
\hline Sem informação & 6 & 50,0 & 33,3 & 16,7 & 0,0 & \\
\hline
\end{tabular}


Tabela 1. Prevalência de (in)segurança alimentar de famílias com crianças assistidas em creches segundo características biológicas das crianças, morbidade e amamentação das crianças, antecedentes maternos e condições socioeconômicas. Paraíba (PE), 2008.

Conclusão

\begin{tabular}{|c|c|c|c|c|c|c|}
\hline \multirow{2}{*}{ Variáveis } & \multirow{2}{*}{ Total $(n=332)$} & \multirow{2}{*}{ Segurança alimentar (\%) } & \multicolumn{3}{|c|}{ Insegurança alimentar (\%) } & \multirow{2}{*}{$p$} \\
\hline & & & Leve & Moderada & Grave & \\
\hline Estatura/ldade & & & & & & $<0,001$ \\
\hline Baixa estatura & 132 & 37,9 & 22,0 & 28,0 & 12,1 & \\
\hline Estatura normal & 190 & 38,9 & 41,1 & 9,5 & 10,5 & \\
\hline Sem informação & 10 & 20,0 & 40,0 & 10,0 & 30,0 & \\
\hline IMC & & & & & & 0,752 \\
\hline Obesidade & 32 & 43,8 & 28,1 & 15,6 & 12,5 & \\
\hline Sobrepeso & 94 & 42,6 & 30,9 & 16,0 & 10,6 & \\
\hline Normalidade & 177 & 37,3 & 33,9 & 17,5 & 11,3 & \\
\hline Baixo peso & 19 & 21,1 & 47,4 & 21,1 & 10,5 & \\
\hline Sem informação & 10 & 20,0 & 40,0 & 10,0 & 30,0 & \\
\hline Cuidados durante a gravidez ${ }^{1}$ & & & & & & 0,160 \\
\hline Bons & 215 & 38,1 & 34,4 & 18,1 & 9,3 & \\
\hline Ruins & 107 & 36,4 & 29,9 & 15,9 & 17,8 & \\
\hline Sem informação & 10 & 50,0 & 50,0 & 0,0 & 0,0 & \\
\hline Condições socioeconômicas & & & & & & 0,038 \\
\hline \multicolumn{7}{|l|}{ Tipo de casa } \\
\hline Própria & 179 & 41,3 & 29,1 & 20,1 & 9,5 & \\
\hline Alugada & 115 & 33,9 & 43,5 & 9,6 & 13,0 & \\
\hline Cedida & 29 & 34,5 & 27,6 & 24,1 & 13,8 & \\
\hline Invadida & 9 & 33,3 & 11,1 & 22,2 & 33,3 & \\
\hline Número de cômodos do domicílio & & & & & & 0,277 \\
\hline$<4$ & 66 & 39,4 & 24,2 & 21,2 & 15,2 & \\
\hline$\geq 4$ & 266 & 37,6 & 35,7 & 15,8 & 10,9 & \\
\hline \multicolumn{7}{|l|}{ Número de pessoas no domicílio } \\
\hline$\geq 6$ & 89 & 31,5 & 40,4 & 14,6 & 13,5 & 0,281 \\
\hline$<6$ & 243 & 40,3 & 30,9 & 17,7 & 11,1 & \\
\hline \multicolumn{7}{|l|}{ Renda familiar per capita ${ }^{2}$} \\
\hline$\geq 2 \mathrm{SM}$ & 2 & 100,0 & 0,0 & 0,0 & 0,0 & $<0,001$ \\
\hline$<2 S M-\geq 1 S M$ & 7 & 85,7 & 14,3 & 0,0 & 0,0 & \\
\hline$<1 S M-\geq 1 / 2 S M$ & 55 & 69,1 & 29,1 & 1,8 & 0,0 & \\
\hline$<1 / 2 S M$ & 266 & 30,0 & 34,6 & 20,7 & 14,7 & \\
\hline Sem informação & 2 & 0,0 & 100,0 & 0,0 & 0,0 & \\
\hline Benefício do Programa Bolsa Família & & & & & & 0,029 \\
\hline Não recebe & 196 & 41,8 & 35,7 & 13,3 & 9,2 & \\
\hline Beneficiário & 136 & 32,4 & 30,1 & 22,1 & 15,4 & \\
\hline
\end{tabular}

Nota: 'Foram consideradas mães com cuidados adequados durante a gravidez aquelas que receberam orientação sobre a amamentação, suplementação com sulfato ferroso, vacina antitetânica, três ou mais consultas pré-natais e que não fizeram uso de cigarro nem de álcool durante o período gestacional; ${ }^{2}$ Considerando o valor do salário-mínimo da época $(R \$ 416,00)$.

IMC: Índice de Massa Corporal; SM: Salário-Mínimo; $p$ : valores de $p$ para o teste estatístico Qui-quadrado; $p$ em negrito denota significância estatística.

$39,7 \%$, baixa estatura, 35,7\%, sobrepeso/obesidade, e 31,7\% das entrevistadas referiram cuidados inadequados durante a gravidez. Foi verifica- do que 19,1\% dos domicílios tinham menos de quatro cômodos e 29,1\%, seis pessoas ou mais. Além disso, a maioria das famílias (94,5\%) tinha 
renda per capita inferior a meio salário-mínimo, e 45,7\% eram beneficiários do Programa Bolsa Família.

\section{Insegurança alimentar}

A prevalência de insegurança alimentar foi de $62,0 \%$, 33,4\% dos domicílios com insegurança alimentar leve, 16,9\% com insegurança alimentar moderada e 11,7\% com insegurança alimentar grave. A distribuição da prevalência de (in)segurança alimentar segundo variáveis biológicas das crianças, morbidade e amamentação das crianças, antecedentes maternos e condições socioeconômicas é apresentada na Tabela 1.

Menores prevalências de insegurança alimentar foram encontradas nos domicílios de crianças amamentadas por mais tempo. Tanto para o aleitamento materno total quanto para o aleitamento materno exclusivo (exceto para insegurança alimentar moderada no caso do aleitamento materno total), foi verificada significância estatística para tal observação. Baixa estatura materna e idade da mãe <20 anos também foram fatores associados à insegurança alimentar familiar.

Piores condições de habitação (tipo de casa) corresponderam às piores situações de insegurança alimentar: as famílias que moram em casas invadidas apresentaram maior proporção de insegurança alimentar. Dentre as famílias com renda mensal per capita menor que meio salário-mínimo, 70,0\% apresentaram insegurança alimentar, sendo $14,7 \%$ na categoria grave (todas as famílias com insegurança alimentar grave estavam no estrato de renda mais baixo). O rendimento médio per capita esteve inversamente associado à si-

Tabela 2. Medidas de Risco (Razão de Chances - RC) e Intervalo de Confiança (IC95\%) para os determinantes de insegurança alimentar de famílias com crianças assistidas em creches. Paraíba (PE), 2008.

\begin{tabular}{|c|c|c|c|c|}
\hline Variáveis & Segurança alimentar & Insegurança alimentar & $\mathrm{RC}$ & IC95\% \\
\hline \multicolumn{5}{|c|}{ Tempo de amamentação total } \\
\hline$\geq 24$ meses & 44 & 48 & 1 & \\
\hline$<24$ meses & 80 & 156 & 1,79 & $1,095-2,917$ \\
\hline \multicolumn{5}{|c|}{ Tempo de amamentação exclusivo } \\
\hline$\geq 6$ meses & 62 & 72 & 1 & \\
\hline$<6$ meses & 62 & 130 & 1,81 & $1,146-2,845$ \\
\hline \multicolumn{5}{|l|}{ Idade da mãe (anos) } \\
\hline$\geq 20$ & 117 & 186 & 1 & \\
\hline$<20$ & 6 & 17 & 1,78 & $0,683-4,650$ \\
\hline \multicolumn{5}{|c|}{ Estatura/ldade da mãe } \\
\hline Estatura normal & 74 & 116 & 1 & \\
\hline Baixa estatura & 50 & 82 & 1,05 & $0,663-1,652$ \\
\hline \multicolumn{5}{|l|}{ Tipo de casa } \\
\hline Própria ou alugada & 113 & 181 & 1 & \\
\hline Outro & 13 & 25 & 1,20 & $0,590-2,442$ \\
\hline \multicolumn{5}{|c|}{ Renda familiar per capita ${ }^{1}$} \\
\hline$\geq 1 / 2 \mathrm{SM}$ & 46 & 18 & 1 & \\
\hline$<1 / 2 \mathrm{SM}$ & 80 & 186 & 5,94 & $3,246-10,877$ \\
\hline \multicolumn{5}{|c|}{ Beneficio do Programa Bolsa Família } \\
\hline Não recebe & 82 & 114 & 1 & \\
\hline Beneficiário & 44 & 92 & 1,5 & 1,136-2,377 \\
\hline
\end{tabular}

Nota: ${ }^{1}$ Considerando o valor do salário-mínimo da época $(R \$ 416,00)$.

SM: Salário-Mínimo; IC95\%: Intervalo de Confiança de 95\%; IC95\% em negrito denota associação estatística. 
tuação de insegurança alimentar, ou seja, quanto menor a renda, maiores as prevalências de insegurança alimentar leve, moderada e grave. A proporção de famílias em insegurança alimentar grave foi estatisticamente maior entre as famílias de menor renda. Ser beneficiário do Programa Bolsa Família mostrou, igualmente, associação estatística com o desfecho.

Os resultados da Tabela 2 apresentam os valores da Razão de Chances e Intervalo de Confiança de $95 \%$ dos determinantes de insegurança alimentar em modelo ajustado. Observa-se que a situação de insegurança alimentar apresenta maior probabilidade de ocorrência nas famílias de menor renda per capita, de crianças com aleitamento materno menor do que 24 meses, e de crianças com amamentação exclusiva menor do que seis meses. A renda familiar per capita esteve fortemente associada à insegurança alimentar das famílias; a renda inferior a meio salário-mínimo aumentou em 5,94 vezes a chance de insegurança alimentar. Os tempos de amamentação total inferior a 24 meses e exclusivo inferior a seis meses aumentaram em quase duas vezes a mesma chance.

\section{DISCUSS Ã O}

Os resultados das PNAD de 2004 e $2009^{18}$ mostraram que a insegurança alimentar é um tema preocupante em nível nacional, com prevalências maiores nas Regiões Nordeste $(53,6 \%$ em 2004 e 46, 1\% em 2009) e Norte $(46,4 \%$ em 2004 e $40,3 \%$ em 2009). Comportamento desigual também foi observado em 2004 para a prevalência da insegurança alimentar grave entre as cinco Regiões brasileiras, sendo 3,1 e 3,5 vezes maior nos domicílios do Norte e do Nordeste respectivamente do que nos domicílios do Sul do País ${ }^{8}$. Para o Estado da Paraíba, os resultados de 2004 apontaram 53,3\% das famílias com insegurança alimentar: 17,4\% leve, 20,8\% moderada e 15,1\% grave ${ }^{8}$. Em 2009, a insegurança alimentar das famílias paraibanas foi de $41,0 \%{ }^{17}$. Estudo realizado em municípios do interior da Paraíba estimou insegurança alimentar em 52,5\% das famílias, sendo 23,6\% leve, 17,6\% moderada e $11,3 \%$ grave $^{4}$. Prevalências de insegurança alimentar um pouco maiores observadas no presente estudo poderiam ser explicadas pela vulnerabilidade socioeconômica e homogeneidade nesse contexto da população estudada.

A renda familiar per capita foi a variável que mostrou maior associação significativa com a insegurança alimentar no presente estudo. Resultados de outros estudos brasileiros em diferentes contextos geográficos ${ }^{3,4,7,19,20}$ confirmam a possibilidade de maiores prevalências de insegurança alimentar acontecerem em famílias com rendimentos menores. A importância da renda familiar per capita na insegurança alimentar das famílias brasileiras também foi constatada na análise dos dados da PNAD de 200421. A disponibilidade de alimentos no Brasil (2960kcal/dia por pessoa), muito acima do recomendado $(1900 \mathrm{kcal} /$ dia por pessoa), e a ingestão de uma alimentação com menos de $1650 \mathrm{kcal} / \mathrm{dia}$ em uma de cada dez pessoas sugerem que o acesso ao alimento é um fator importante na determinação da insegurança alimentar no País ${ }^{22}$. No Brasil, o acesso de toda a população a alimentos de qualidade e em quantidade suficiente depende, predominantemente, da renda que o indivíduo ou sua família dispõe e dos preços a que tais alimentos são vendidos ${ }^{1}$. Segundo Batista Filho ${ }^{23}$, a baixa renda familiar, o desemprego, o subemprego e as oscilações bruscas de preços são fatores estruturais e conjunturais que situam 45 milhões de brasileiros abaixo da linha de pobreza e indigência.

A importância da condição socioeconômica como determinante da insegurança alimentar também tem sido observada em outros estudos ${ }^{3,19,20}$, que verificaram sua associação com outras variáveis, como a escolaridade do chefe da família ou pessoa de referência ${ }^{3,21}$, o número de moradores no domicílio ${ }^{3}$, a condição do domicílio ${ }^{19}$ e a cobertura de saneamento básico ${ }^{20,21}$. Neste estudo, o benefício do Programa Bolsa Família foi condição socioeconômica importante na determinação da insegurança alimentar. As 
maiores prevalências de insegurança alimentar moderada e grave nos casos das famílias beneficiárias do Programa Bolsa Família do presente estudo, sugerindo o direcionamento adequado dos recursos do Programa, foi fato também constatado mediante análises realizadas com dados nacionais ${ }^{8,24}$. No Brasil, segundo os dados da PNAD de 2004, a importância dos programas de transferência de renda na redução da insegurança alimentar das famílias foi constatada em modelo estatístico que mostrou chance maior de $8 \%$ de segurança alimentar para cada $R \$ 10,00$ de acréscimo nos valores transferidos ${ }^{25}$.

O aleitamento materno inferior aos 24 meses e o aleitamento materno exclusivo inferior a seis meses foram também condições que mais aumentaram a chance das famílias estarem em situação de insegurança alimentar. Estudo baseado em dados de âmbito nacional, considerando crianças menores de dois anos, mostrou associação entre o aleitamento materno e a situação de segurança alimentar familiar nas crianças maiores de 12 meses $^{26}$. Nenhum outro estudo abordando o tema foi desenvolvido em população de crianças brasileiras. Pressupõe-se, assim, a necessidade de estudos que analisem a possibilidade do aleitamento materno (total e exclusivo) beneficiar a situação de segurança alimentar, uma vez que: i) o tempo em que a mãe amamenta a criança pode estar influenciado pelo seu nível de escolaridade, fator que pode influenciar a probabilidade de ocorrência de insegurança alimentar ${ }^{3,7}$; ii) o leite materno, além de proteger a criança contra infecções, constitui um alimento disponível e barato que pode beneficiar a segurança alimentar das famílias de menor poder aquisitivo, ao contrário do desmame precoce que pode implicar custo maior para alimentar a criança27; iii) o aleitamento materno pode proteger crianças em condições adversas produzidas por insegurança alimentar e nutricional26.

A inexistência de estudos no Brasil sobre vulnerabilidade à insegurança alimentar entre famílias com crianças atendidas em creches torna oportunos e relevantes os resultados apresentados neste estudo. Considerando que as crianças recebem alimentação na creche, respostas afirmativas e altas frequências para os itens de maior gravidade e relativos às crianças da EBIA não seriam esperadas. Assim, as inesperadas altas prevalências de insegurança alimentar grave que foram encontradas devem servir de referência para futuros estudos e para o monitoramento e avaliação de políticas públicas de combate à fome.

A renda familiar per capita inferior a meio salário-mínimo foi o fator que mais contribuiu com a insegurança alimentar nessas famílias, sugerindo comprometimento do acesso à alimentação. A dificuldade de acesso é reforçada pela associação entre o benefício do Programa Bolsa Família e a insegurança alimentar. Essa alusão advém do caráter emergencial do benefício para enfrentar situações de insegurança alimentar de segmentos da sociedade com dificuldade de acesso aos alimentos por restrição de renda. Assim, patenteamos a importância da transferência de renda do Programa Bolsa Família na segurança alimentar e familiar. O impacto do Programa Bolsa Família melhora com a adequada focalização e estende-se a outras esferas, como saúde e educação, quando as condicionalidades são cumpridas.

\section{COLABORADORES}

D FIGUEROA PEDRAZA contribuiu para a concepção e delineamento; coleta, análise e interpretação dos dados; redação, revisão e aprovação final do artigo. D QUEIROZ contribuiu para a análise e interpretação dos dados; redação, revisão e aprovação final do artigo. TN MENEZES contribuiu na interpretação dos dados; redação, revisão crítica e aprovação final do artigo.

\section{REFERÊ NCIAS}

1. Nascimento AL, Andrade SLLS. Segurança alimentar e nutricional: pressupostos para uma nova cidadania? Ciênc Cult. 2010; 62(4):34-8.

2. Pereira RA, Santos LMP. A dimensão da insegurança alimentar. Rev Nutr. 2008; 21(Supl):7-13.

3. Salles-Costa R, Pereira RA, Vasconcellos MTL, Veiga GV, Marins VMR, Jardim BC, et al. Associação entre 
fatores socioeconômicos e insegurança alimentar: estudo de base populacional na Região Metropolitana do Rio de Janeiro, Brasil. Rev Nutr. 2008; 21(Supl):99-109.

4. Vianna RPT, Segall-Corrêa AM. Insegurança alimentar das famílias residentes em municípios do interior do Estado da Paraíba, Brasil. Rev Nutr. 2008; 21(Supl):111-22.

5. Pérez-Escamilla R, Segall-Corrêa, AM. Food insecurity measurement and indicators. Rev Nutr. 2008; 21(Supl):15-26.

6. Radimer KL, Olson CM, Greene JC, Campbel CC, Habitch JP. Understanding hunger and developing indicators to assess it in women and children. J Nutr Educ. 1992; 24(1 Suppl):36-45.

7. Favaro T, Ribas DLB, Zorzatio JR, Segall-Corrêa, AM, Panigassi G. Segurança alimentar em famílias indígenas Térena, Mato Grosso do Sul, Brasil. Cad Saúde Pública. 2007; 23(4):785-93.

8. Instituto Brasileiro de Geografia e Estatística. Pesquisa nacional por amostra de domicílios: segurança alimentar 2004. Rio de Janeiro: IBGE; 2006.

9. Figueroa Pedraza D. Grupos vulnerables y su caracterización como criterio de discriminación de la seguridad alimentaria y nutricional en Brasil. Rev Bras Saúde Matern Infant. 2005; 5(3):367-75.

10. Oliveira EFT, Grácio MCC. Análise a respeito do tamanho de amostras aleatórias simples: uma aplicação na área de ciência da informação. Rev Ciênc Inf. 2005; 6(3):1-11.

11. Sociedade Civil Bem-Estar Familiar no Brasil. Pesquisa Nacional sobre Demografia e Saúde: 1996. $2^{a}$ ed. Rio de Janeiro; 1999.

12. World Heath Organization. Physical status: The use and interpretation of anthropometry: Report of an expert committee. Geneva: WHO; 1995. WHO Technical Report Series, 854.

13. Onis M, Onyango AW, Van Den Broeck J, Chumlea WC, Martorell R. Measurement and standardization protocols for anthropometry used in the construction of a new international growth reference. Food Nutr Bull. 2004; 25(Suppl 1):15-27.

14. World Health Organization. Global database on child growth and malnutrition. Geneva: WHO; 2007 [cited 2010 Mar 5]. Available from: <http:// www.who.int/nutgrowthdb/index.html>.

15. National Center for Health Statistics, Centers for Disease Control and Prevention. National Health and Nutrition Examination Survey. New York: CDC; 2000 [cited 2010 Mar 13]. Available from: <http:// www.cdc.gov/growtharts>.
16. Maletta H, Gómez R. Seguridad amimentaria: medición y métodos. FAO; 2004.

17. Segall-Corrêa AM, Pérez-Escamilia R, Sampaio MFA, Marin-Leon L, Panigassi G, Maranha LK. Acompanhamento e avaliação da segurança alimentar de famílias brasileiras: validação de metodologia e de instrumento de coleta de informação: urbano/rural. 2004. Campinas: Unicamp; 2004 [acesso 2010 abr 1]. Disponível em: <http://www. opas.org.br/sistema/arquivos/vru_unic.pdf>.

18. Instituto Brasileiro de Geografia e Estatística. Pesquisa Nacional por Amostra de Domicílios. Segurança Alimentar 2004/2009. Rio de Janeiro: IBGE; 2010.

19. Santos JV, Gigante DP, Domingues MR. Prevalência de insegurança alimentar em Pelotas, Rio Grande do Sul, Brasil, e estado nutricional de indivíduos que vivem nessa condição. Cad Saúde Pública. 2010; 26(1):41-9.

20. Panigassi G. Inquérito populacional sobre a percepção da segurança alimentar intrafamiliar no município de Campinas, SP [doutorado]. Campinas: Unicamp; 2005.

21. Hoffmann R. Determinantes da insegurança alimentar no Brasil: análise dos dados da PNAD de 2004. Segur Aliment Nutr. 2008; 15(1):49-61.

22. Leitão Júnior NA, Nehme VGF. O desafio da sustentabilidade: um breve ensaio sobre os limites da realidade. Observatorium. 2009; 1(1):197-210.

23. Batista Filho M. Projeto fome zero: a importância da divulgação científica dos seus resultados. Rev Bras Saúde Matern Infant. 2003; 3(1):7-8.

24. Brasil. Ministério da Saúde. Pesquisa nacional sobre demografia e saúde da criança e da mulher. Brasília: MS; 2008.

25. Segall-Corrêa AM, Marin-Leon L, Helito H, PérezEscamilla R, Santos LMP, Paes-Sousa R. Transferência de renda e segurança alimentar no Brasil: análise dos dados nacionais. Rev Nutr. 2008; 21(Supl):39-51.

26. Gomes GP, Gubert MB. Breastfeeding in children under 2 years old and household food and nutrition security status. J Pediatr. 2012; 88(3):279-82.

27. Brasil. Ministério da Saúde. Dez passos para uma alimentação saudável: guia alimentar para crianças menores de dois anos: álbum seriado. $2^{\text {a }}$ ed. Brasília: MS; 2003. 
